\title{
Primary Duodenal Adenocarcinoma: Uncommon Tumor, Uncommon Presentation. A Case Report and Review of the Literature
}

This article was published in the following Dove Press journal: International Medical Case Reports Journal

\author{
Shital Khanal' \\ Utsav Joshi (iD) ' \\ Adheesh Bhattarai (iD ${ }^{\prime}$ \\ Vishakha Agrawal' \\ Gita Sayami (D) ${ }^{2}$ \\ Ramesh Singh Bhandari \\ 'Department of Gl and General Surgery, \\ Institute of Medicine, Tribhuvan \\ University, Kathmandu, Nepal; \\ ${ }^{2}$ Department of Pathology, Institute of \\ Medicine, Tribhuvan University, \\ Kathmandu, Nepal
}

Introduction: The symptoms of primary duodenal adenocarcinoma, which is a rare but aggressive tumor, are vague and nonspecific and often result in a delayed diagnosis or misdiagnosis. This results in a tumor being diagnosed at an advanced stage when it becomes unresectable secondary to local and distant spread.

Case Presentation: A 64-year-old Nepalese female presented to our hospital with epigastric pain, anorexia, and significant weight loss that developed over two-and-a-half months. Upper gastrointestinal endoscopy showed an ulceroproliferative growth in the first part of the duodenum with no features of duodenal stenosis. Contrast-enhanced computed tomography of the abdomen revealed heterogeneously enhancing, circumferential, asymmetrical thickening in the first part of the duodenum and multiple liver metastases. Biopsy of the mass revealed features suggestive of moderately differentiated adenocarcinoma of the duodenum. She was managed with palliative care during her hospital stay. The unique presentation in our case was that the tumor did not cause stenosis and the patient could consume food till the last day of her life.

Conclusion: In patients with primary duodenal adenocarcinoma, the non-stenotic lesion is also a possibility. Clinicians should always maintain a high degree of suspicion to avoid the delay in diagnosis or misdiagnosis.

Keywords: primary duodenal adenocarcinoma, non-stenotic lesion, first part, duodenum

\section{Introduction}

Primary duodenal adenocarcinoma (PDA) is an uncommon entity, accounting for $0.3-1 \%$ of all gastrointestinal malignancies. ${ }^{1-3}$ Most PDA originate in the second part of the duodenum, with the first part being the least common site of origin. ${ }^{4}$ Patients often present with vague and nonspecific symptoms that may divert suspicion towards a more benign diagnosis and preclude relevant investigations. ${ }^{5}$ Owing to the low incidence and the lack of specificity of clinical presentations, PDA poses a significant diagnostic challenge. As a result, patients are misdiagnosed or diagnosed at a late stage of the disease, by which time, resection of the tumor becomes incompatible with the scale of invasion and metastasis. ${ }^{6}$ Even though the optimal surgical procedure remains controversial, many authors agree that a radical surgical approach that achieves complete tumor resection is of utmost benefit whenever feasible., ${ }^{3,7}$

We report an interesting case of a 64-year-old female patient diagnosed to have adenocarcinoma involving the first part of the duodenum with no signs of intestinal obstruction.
Maharajgunj Medical Campus, Institute of Medicine, Tribhuvan University, PO Box

1524, Kathmandu, Nepal

Tel +9779849726254

Fax +977 | 442377।

Email utsavjoshi.uh@gmail.com 


\section{Case Presentation}

A 64-year-old smoker and non-alcoholic Nepalese female presented to the outpatient department of a university hospital with a complaint of epigastric pain for two-and-a-half months. This was not associated with abdominal distension, nausea or vomiting, and her bowel habits were normal. There was no history of fever, melena, hematemesis, and jaundice. However, her epigastric pain was associated with anorexia and loss of approximately six $\mathrm{kg}$ of weight in the preceding two months. She had undergone vaginal hysterectomy six years back for uterovaginal prolapse.

On examination, her general condition was fair, and vital signs were stable. There was no icterus, and she was afebrile. Abdominal examination revealed mild tenderness in the epigastric region. However, there was no guarding, rigidity, or rebound tenderness in any quadrant of the abdomen. Bowel sounds were heard all over the abdomen. The rest of the systemic examinations were normal.

Baseline blood investigations showed anemia, normal blood sugar level, liver function tests, renal function tests, and serology. Upper gastrointestinal (UGI) endoscopy showed an ulceroproliferative growth with elevated and irregular margin in the first part of the duodenum and a deformed pylorus. Despite this, the mass did not result in the constriction of the duodenal lumen. A biopsy of the mass was also obtained in the same sitting and sent for histopathological evaluation.

Following UGI endoscopy, contrast-enhanced computed tomography (CECT) of the abdomen was planned for further evaluation of the mass. The CECT scan showed heterogeneously enhancing, circumferential, asymmetrical thickening in the first part of the duodenum and multiple liver metastases (Figures 1 and 2). Biopsy of the mass revealed fragments of duodenal tissue with infiltrating malignant cells arranged in a glandular pattern and scattered singly (Figures 3 and 4). Tumor cells were moderately pleomorphic, had increased nucleus to cytoplasmic ratio, and showed vesicular nucleus with prominent nucleoli. Intervening stroma showed infiltration mixed with inflammatory cells. Thus, a final diagnosis of moderately differentiated adenocarcinoma of the duodenum was established.

Even though the treatment of choice is a radical surgical resection, we did not perform surgery in this patient because the tumor was already at an advanced stage. The patient and her family members were counseled about palliative chemotherapy; however, having understood the prognosis of her disease, they denied chemotherapy. She received supportive care during her stay in the hospital. A unique finding in our patient was that she could consume food until the last day of her life before she passed away 49 days after her admission to the hospital.

\section{Discussion}

This case report discusses a rare form of gastrointestinal tumor and its unique presentation in our patient. There are very few reported cases of PDA in the first part of the duodenum that presents without stenosis and even less so from Nepal. In this regard, this case report may help us broaden our knowledge and understanding of the subject.

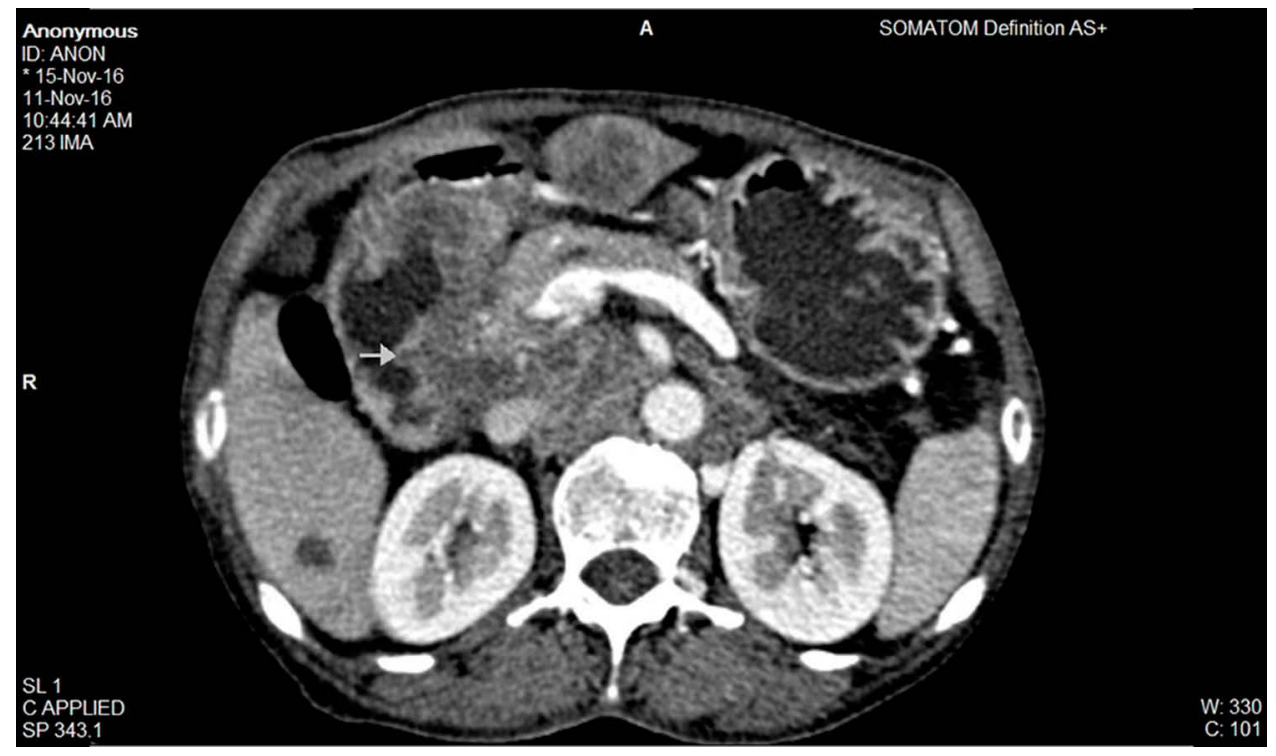

Figure I CECT image of the abdomen - the image shows asymmetrical thickening of the duodenal wall (arrow). 


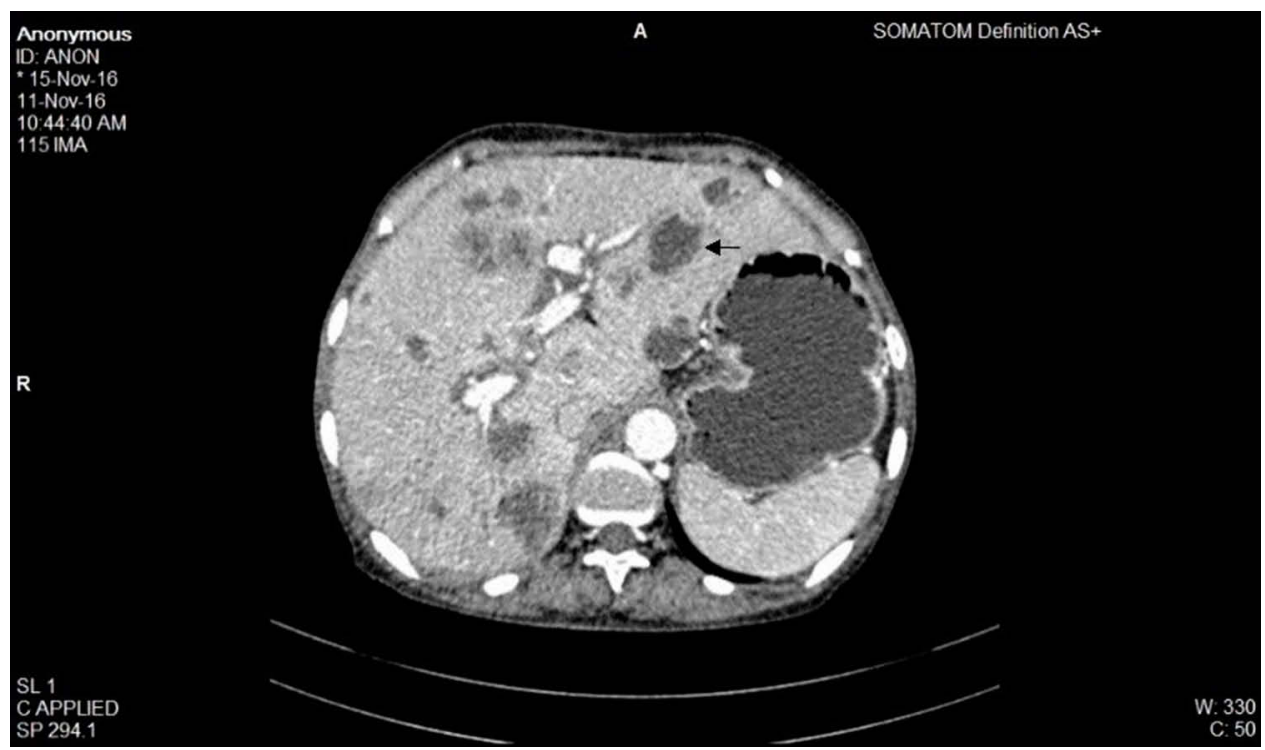

Figure 2 CECT image of the abdomen - the image shows multiple liver metastases (arrow).

The small bowel is the least common site for primary malignancy in the gastrointestinal tract. Data from the Surveillance, Epidemiology, and End Results (SEER) over 15 years from 1992 to 2006 shows that the average annual age-adjusted incidence rates of small bowel carcinoma in the US population are 1.45 and 1.00 per 100,000 population for males and females respectively. ${ }^{6,8}$ As the data suggests, men have a higher incidence of PDA than women and there is an increased incidence of this malignant tumor after the age of 40 years. $^{9}$ Adenocarcinoma represents the major bulk of small bowel cancer, followed by carcinoid tumor, lymphoma, and leiomyosarcoma. ${ }^{2}$ However, when taken into a broader context of all gastrointestinal malignancies, PDA is still a relatively rare tumor. Most PDA originate in the second part of the duodenum, followed by the third, fourth, and first part with an incidence of 50-75\%, 14-33\%, $5-17 \%$, and $1-15 \%$ respectively. ${ }^{7}$

The etiology of PDA has not been clearly identified, but certain groups of people are at a higher risk of developing PDA in their lifetime. These include patients with Familial Adenomatous Polyposis, Crohn's disease, Celiac disease, Peutz-Jeghers syndrome, and Hereditary Non-Polyposis Colorectal Cancer. ${ }^{8,10}$ In the study by Dabaja et al, abdominal pain and intestinal obstruction were by far the most common

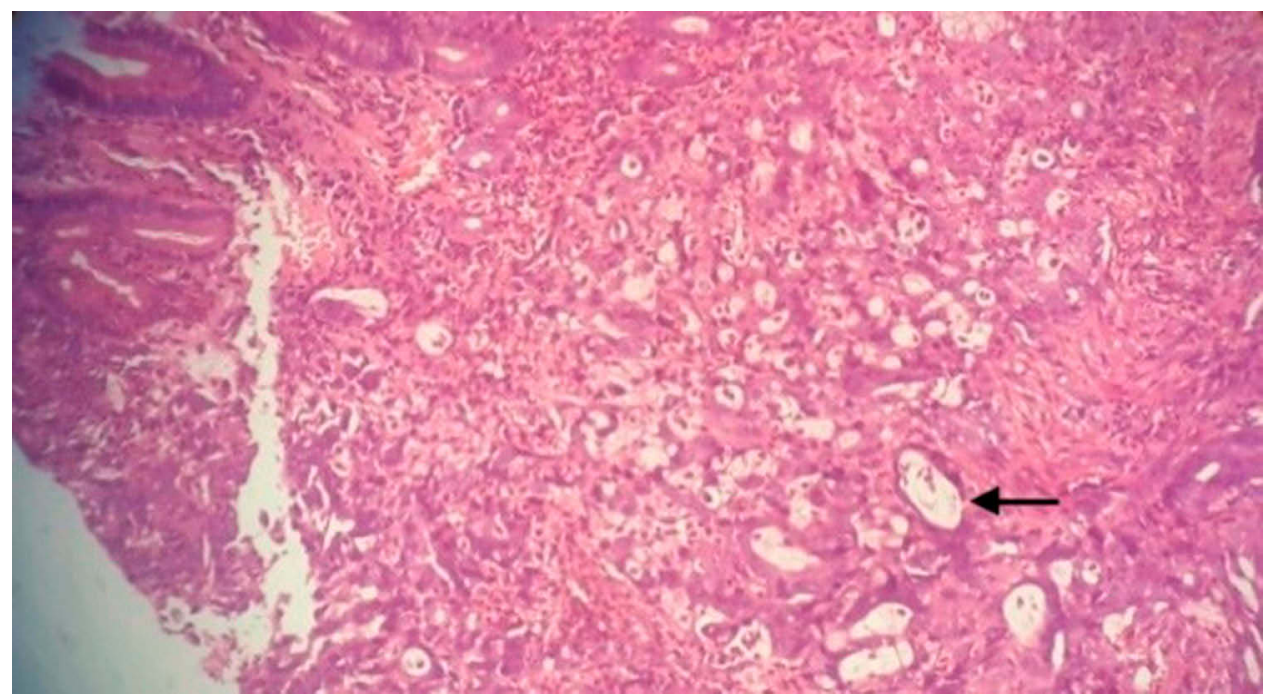

Figure 3 Histopathological image of the tumor - the image shows malignant cells arranged in tubules (arrow) and solid nests. H\&E (xI00). 


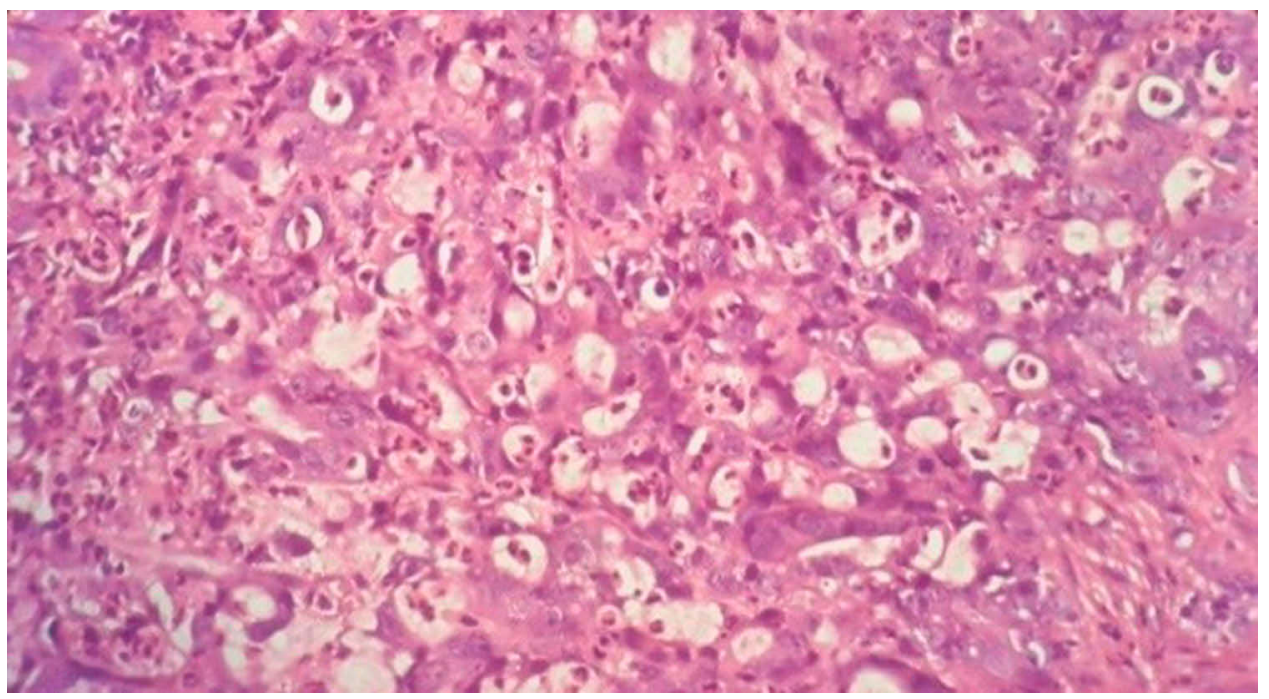

Figure 4 Histopathological image of the tumor - the image shows tumor cells with moderate amount of cytoplasm and round to oval vesicular nuclei and prominent nucleoli. H\&E (x400).

clinical presentations in patients with PDA, followed by bleeding. ${ }^{10}$ Even though intestinal obstruction is a common presentation secondary to the stenotic lesion in the duodenum, it was absent in our patient. Patients can also present with nausea, vomiting, jaundice, fatigue, weakness, anemia, and weight loss. ${ }^{4,7}$ The problem with establishing the diagnosis of PDA is twofold. First, patients may not show symptoms until tumors become sufficiently large or reach a very advanced stage. Second, the initial symptoms are highly nonspecific and vague. Because of these reasons, an average delay of 2-15 months from the onset of symptoms to the final diagnosis of PDA is common. ${ }^{5,11}$ Besides, these nonspecific symptoms are often mistaken for some other conditions, and this misdiagnosis rate of PDA was a whopping $56 \%$ in a study by Zhou et al. ${ }^{12}$

Esophagogastroduodenoscopy and barium contrast studies remain the first-line diagnostic modalities for the evaluation of PDA. ${ }^{2,7}$ Endoscopy allows direct visualization of the mucosal surface of the duodenum and biopsy of the mass. Barium studies show intraluminal mass lesions, stenosis or obstruction, and help to rule out other causes. ${ }^{4} \mathrm{CECT}$ must be performed in all biopsy-confirmed cases to stage cancer, determine its resectability, and plan necessary intervention. ${ }^{2,5}$ Malignant lesions typically appear as an exophytic or intramural mass with central necrosis and ulceration, while benign lesions appear entirely intraluminal. ${ }^{7}$ Carcinoembryonic Antigen (CEA) and Carbohydrate Antigen 19-9 (CA 19-9) are elevated in 40-50\% of the patients with ampullary and non-ampullary duodenal tumors; however, it is neither highly sensitive nor specific to have any significant role in diagnosis. ${ }^{11}$
Many factors should be considered before choosing the specific intervention for PDA- location of the tumor, tumor staging, availability of expert facilities, and condition of the patient. ${ }^{11}$ Radical resection of the tumor remains the first-choice for curability. ${ }^{2}$ As the tumor has nonspecific symptoms and poorly defined natural history, it is usually diagnosed at an advanced stage when the possibility of curative resection is almost non-existent. ${ }^{1}$ Excluding these non-feasible cases, pancreaticoduodenectomy remains the preferred surgical option for adenocarcinoma of the first and second part of the duodenum and segmental resection is often reserved for adenocarcinoma of the third and fourth part. ${ }^{5,11}$ Pancreaticoduodenectomy is considered a superior surgical intervention than segmental resection because resection margin is wider and regional lymph nodes clearance is more extensive. ${ }^{3}$

Kawahira et al retrospectively studied the outcomes in 21 patients with PDA and found that tumor size, tumor depth, tumor differentiation, and lymph node metastasis had a significant negative impact on survival. The same study determined the mean survival rates of the patients with PDA at 1,3 , and 5 years after surgery to be $66.2 \%$, $48.2 \%$, and $38.6 \%$ respectively. ${ }^{3}$ In a large-scale study of 217 patients by Dabaja et al, the 5-year overall survival rate was significantly higher in the surgical group compared to those who did not undergo surgery. ${ }^{10}$

In the presented case, the patient complained of vague symptoms like epigastric pain, anorexia, and weight loss. Further evaluation with endoscopy, biopsy, and CECT established the diagnosis of PDA. However, the patient did not 
have any clinical manifestation of intestinal obstruction. Moreover, the investigation did not reveal any stenosis in the first part of the duodenum, which was the site of tumor growth. The rare presentation of the tumor in our patient is brought into sharper focus when one considers the fact that the first part of the duodenum is the least common site of tumor occurrence and that stenosis with features of obstruction is very common with PDA. The uncommon presentation most probably resulted in delayed diagnosis and allowed the tumor to reach an advanced stage. Hence, the patient was only given palliative care.

\section{Conclusion}

Primary adenocarcinoma of the duodenum is a rare malignancy, even more so when presenting without stenosis. Even though the clinical manifestations are nonspecific, clinicians should always maintain a high degree of suspicion to avoid the delay in diagnosis or misdiagnosis.

\section{Abbreviations}

PDA, Primary Duodenal Adenocarcinoma; UGI, Upper Gastrointestinal; CECT, Contrast-Enhanced Computed Tomography; SEER, Surveillance, Epidemiology, and End Results; CEA, Carcinoembryonic Antigen; CA, Carbohydrate Antigen.

\section{Consent for Participation and Publication}

Written informed consent was obtained from the patient for the publication of this case report and any accompanying images. A copy of the written consent will be made available for review upon request.

\section{Author Contributions}

SK and UJ compiled the case and drafted the initial manuscript. $A B$ and VA did the initial literature review. GS performed the histological examination of the tumor. RSB did the clinical diagnosis, investigations, and treatment of the case. All authors made substantial contributions to conception and design, acquisition of data, or analysis and interpretation of data; took part in drafting the article or revising it critically for important intellectual content; gave final approval of the version to be published; and agree to be accountable for all aspects of the work.

\section{Disclosure}

The authors report no conflicts of interest in this work.

\section{References}

1. You HS, Hong JW, Yun EY, et al. Primary non-ampullary duodenal adenocarcinoma: a single-center experience for 15 years. Korean J Gastroenterol. 2015;66(4):194-201. doi:10.4166/kjg.2015.66.4.194

2. Lee CC, Ng WK, Lin KW, Lai TW, Li SM. Adenocarcinoma of the duodenum. Hong Kong Med J. 2008;14(1):67.

3. Kawahira H, Miura F, Saigo K, et al. Survival predictors of patients with primary duodenal adenocarcinoma. Int Surg. 2011;96 (2):111-116. doi:10.9738/1381.1

4. Cloyd JM, George E, Visser BC. Duodenal adenocarcinoma: advances in diagnosis and surgical management. World J Gastrointest Surg. 2016;8(3):212. doi:10.4240/wjgs.v8.i3.212

5. Bandi M, Scagliarini L, Anania G, Pedriali M, Resta G. Focus on the diagnostic problems of primary adenocarcinoma of the third and fourth portion of the duodenum. Case report. Il Giornale di chirurgia. 2015;36(4):183.

6. Tangkittikasem N, Boonyaarunnate T, Aswakul P, Kachintorn U, Prachayakul V. Clinical, radiologic, and endoscopic manifestations of small bowel malignancies: a first report from Thailand. Asian Pac $J$ Cancer Prev. 2015;16(18):8613-8618. doi:10.7314/APJCP.2015. 16.18.8613

7. Usuda D, Hashimoto Y, Muranaka E, Okamura H, Kanda T, Urashima S. Primary duodenal adenocarcinoma without stenosis: a case report with a brief literature review. Case Rep Oncol. 2014;7 (2):444-451. doi:10.1159/000365186

8. Pan SY, Morrison H. Epidemiology of cancer of the small intestine. World J Gastrointest Oncol. 2011;3(3):33. doi:10.4251/wjgo.v3.i3.33

9. Curado MP, Edwards B, Shin HR, et al. Cancer Incidence in Five Continents. Vol. IX. IARC Press, International Agency for Research on Cancer; 2007.

10. Dabaja BS, Suki D, Pro B, Bonnen M, Ajani J. Adenocarcinoma of the small bowel: presentation, prognostic factors, and outcome of 217 patients. Cancer. 2004;101(3):518-526. doi:10.1002/cncr.20404

11. Wang Z, Ding Z, Huang S, Zhong S. Experience in clinical diagnosis and treatment of duodenal tumors. Mol Clin Oncol. 2016;5 (6):731-739. doi:10.3892/mco.2016.1061

12. Zhou ZW, Wan DS, Chen G, Chen YB, Pan ZZ. Primary malignant tumor of the small intestine. World J Gastroenterol. 1999;5(3):273. doi:10.3748/wjg.v5.i3.273

International Medical Case Reports Journal

Dovepress

\section{Publish your work in this journal}

The International Medical Case Reports Journal is an international, peer-reviewed open-access journal publishing original case reports from all medical specialties. Previously unpublished medical posters are also accepted relating to any area of clinical or preclinical science. Submissions should not normally exceed 2,000 words or 4

published pages including figures, diagrams and references. The manuscript management system is completely online and includes a very quick and fair peer-review system, which is all easy to use. Visit http://www.dovepress.com/testimonials.php to read real quotes from published authors. 